\title{
Simultaneous profiling of chromatin accessibility and methylation on human cell lines with nanopore sequencing
}

\author{
Authors: \\ Simpson ${ }^{2,4}$, Fritz J. Sedlazeck ${ }^{3}$, Winston Timp ${ }^{1}$ \\ 1. Johns Hopkins University Department of Biomedical Engineering \\ 2. Ontario Institute for Cancer Research, Toronto, Ontario, Canada \\ 3. Human Genome Sequencing Center, Baylor College of Medicine \\ 4. University of Toronto Department of Computer Science
}

Isac Lee ${ }^{1}$, Roham Razaghi ${ }^{1}$, Timothy Gilpatrick ${ }^{1}$, Michael Molnar ${ }^{2}$, Norah Sadowski ${ }^{1}$, Jared T.

\begin{abstract}
Understanding how the genome and the epigenome work together to control gene transcription has applications in our understanding of diseases such as human cancer. In this study, we combine the ability of NOMe-seq to simultaneously evaluate CpG methylation and chromatin accessibility, with long-read nanopore sequencing technology, a method we call nanoNOMe. We generated $>60 \mathrm{~Gb}$ whole-genome nanopore sequencing data for each of four human cell lines (GM12878, MCF-10A, MCF-7, MDA-MB-231) including repetitive regions inaccessible by short read sequencing. Using the long reads, we find that we can observe phased methylation and chromatin accessibility, large scale pattern changes, and genetic changes such as structural variations from a single assay.
\end{abstract}

\section{INTRODUCTION}

Many human diseases, including all forms of neoplasia, result from aberrant gene regulation through mechanisms including genetic mutation, altered signal transduction, and epigenetic alteration. Healthy cells use tight control of the epigenome to modulate active transcription of genes through the coordination of numerous signals including methylation of $\mathrm{CpG}$ dinucleotides, chromatin accessibility, and nuclear organization. The epigenome is highly mutable, changing dynamically in response to external stimuli, which can result in epigenetic variation among phenotypically and genetically homogeneous populations. This feature is especially evident when comparing tissue-paired normal and cancer samples ${ }^{1}$. Normal tissue commonly has well-defined epigenetic signatures, in contrast to transformed cells wherein cancer epigenetics is much more varied from sample to sample ${ }^{1}$, and even from cell to cell ${ }^{2}$.

With the proliferation of DNA sequencing technologies, methods have been developed for examining nuclear organization, protein binding site occupancy, chromatin accessibility, and methylation state. Many of these methods rely on the vulnerability of accessible chromatin to enzymatic treatment, e.g. DNAse-seq ${ }^{3}$, ATAC-seq $^{4}$. One of these methods, NOMe-seq ${ }^{5}$, labels genomic regions in a nucleosome-depleted, accessible state using an exogenous GpC methyltransferase. Combined with bisulfite conversion, this method permits simultaneous 
evaluation of the endogenous cytosine methylation as well as nucleosome occupancy. However, these methods do not directly interrogate the DNA strand, and the reads are typically too short to provide information about the regional context of the DNA.

The long reads possible with nanopore sequencing provide a deeper level of insight, allowing investigation of long-range patterns on individual DNA molecules. We and others have previously shown that endogenous CpG methylation can be accurately called with nanopore data $^{6,7}$. By extending this model to include the non-native $\mathrm{GpC}$ modifications, we are able to adapt the NOMe-seq workflow to the nanopore platform. We then take advantage of the long read lengths $(>10 \mathrm{~kb})$ generated by nanopore sequencing to read the CpG methylation and chromatin accessibility across stretches of genomic regions at the single molecule level. Using this approach we have simultaneously determined phased patterns of native methylation and chromatin accessibility in four different human cell lines.

\section{RESULTS}

\section{Nanopore GpC methylation calling}

We previously developed a software tool, nanopolish, which can measure CpG methylation from nanopore sequencing ${ }^{6}$. Specifically, nanopolish employs a hidden Markov Model (HMM) to detect cytosine methylation based on electrical current signatures (events) corresponding to groups of nucleotide sequences (k-mers). The HMM uses a table of event level distributions characteristic to every k-mer, termed a pore model, to predict the methylation state of k-mers. The CpG methylation pore model was generated from sequencing data of DNA enzymatically methylated by M.Sssl at $>95 \%$ of all CpG locations as previously described ${ }^{6}$. The methylation caller outputs log-likelihood ratios for the probability of methylation at a given k-mer, and a threshold is applied to determine the binary value of methylation at single-molecule resolution. To expand the nanopolish algorithm to detect cytosine methylation at GpC contexts, we generated a new training set using combinations of M.Sssl (CpG methyltransferase) and M. CviPI (GpC methyltransferase) on unmethylated (PCR amplified) Escherichia coli (E. coli) genomic DNA (see Methods). This resulted in samples with $\mathrm{CpG}, \mathrm{GpC}$, and both $\mathrm{CpG}$ and $\mathrm{GpC}$ methylation as well as an unmodified negative control. The methylation samples were sequenced on Oxford Nanopore Technologies (ONT) MinIONs using the sequencing library preparation kit by ligation (LSK-SQK109). The resulting raw data was basecalled using guppy and aligned to the $E$. coli reference genome.

We extracted the event signals and compared the distributions in current for a set of k-mers containing combinations of the methylation motifs. In k-mers that contained motifs for GpC methylation, we observed that the $\mathrm{GpC}$ methylated samples had clear shifts in event level distributions in comparison to unmethylated samples (Fig. 1a). We further observed that in some k-mers that contain both $\mathrm{CpG}$ and $\mathrm{GpC}$ motifs, the three methylated samples had different shifts in current, indicating that $\mathrm{CpG}$ and $\mathrm{GpC}$ methylation could be detected simultaneously in some contexts. The sequencing data from the $\mathrm{GpC}$ methylation sample was used to train the pore model for $\mathrm{GpC}$ methylation detection. 
To benchmark GpC methylation detection, we tested methylation pore models on completely methylated and unmethylated samples of genomic DNA from GM12878 human lymphoblast cell line. As with E. Coli samples, we generated a completely unmethylated sample of GM12878 gDNA from whole genome PCR-amplification, then treated this with either M. SssI (CpG) or M. CviPI (GpC) to generate the methylated samples. The extent of methylation was validated via low coverage whole genome bisulfite sequencing, using $\sim 2 \mathrm{Mb}$ of sequencing data per condition, which confirmed that the methylated samples were methylated at $>95 \%$ of all detected motifs (Supplementary Table 1). These same validated samples were used as the truth sets for unmethylated, $\mathrm{CpG}$ methylated, and $\mathrm{GpC}$ methylated events. To assess the performance of methylation detection, we generated receiver operating characteristic (ROC) curves by applying a range of thresholds to bin methylation statuses (Fig. 1b) as we did previously ${ }^{6}$. Using a log-likelihood ratio of 2.5 as the threshold for calling methylation (where a value $<-2.5$ is unmethylated, $>2.5$ is methylated, and $(-2.5,2.5)$ is not called), we called $95 \%$ of CpGs as methylated in the $72 \%$ of all possible CpG k-mers and $97 \%$ of GpCs as methylated in $89 \%$ of all possible GpC k-mers.(Supplementary Fig. 1a). Both the CpG and GpC models had high area under the curve (AUC) of the ROC curve, confirming the applicability of the models. It should again be emphasized that the bisulfite sequencing data indicated incomplete ( $96-98 \%)$ enzymatic methylation in this sample, so this is a conservative estimate of our accuracy. We used this threshold of 2.5 for subsequent methylation detection.

\section{Chromatin and DNA methylation profiling with NanoNOMe}

We then adapted the existing NOMe-seq protocol ${ }^{5}$ to profile chromatin state for use with nanopore sequencing, terming this modified method nanoNOMe (Fig. 2a). Because nanopore sequencing discriminates methylated cytosines directly, bisulfite conversion and PCR amplification are unnecessary. However, to preserve the modifications, we cannot amplify the DNA requiring a higher (1-2ug) initial amount of DNA as input. Briefly, intact nuclei were extracted from cells by gentle lysis, followed by methylation with $\mathrm{GpC}$ methyltransferase. The methylation treatment of intact nuclei results in $\mathrm{GpC}$ methylation only at unoccupied, open regions of the genome (Fig. 2a). After purification of DNA from these nuclei by phenol:chloroform extraction and ethanol precipitation, we performed ligation-based library preparation for nanopore sequencing (ONT). After sequencing, basecalling, and alignment, we applied our $\mathrm{GpC}$ methylation model to detect $\mathrm{GpC}$ methylation in addition to detecting $\mathrm{CpG}$ methylation using the existing model. Subsequently, methylation at cytosines in a $\mathrm{GCH}$ context were used as a measure of chromatin accessibility and cytosines in a HCG context were used as measures of endogenous methylation, and methylation measurements in GCG cytosines were excluded from analysis. In describing GpCs state, a methylated GpC was interpreted as an accessible mark, and unmethylated as inaccessible.

We performed nanoNOMe on the GM12878 lymphoblast cell line, chosen because it has been well-characterized in previous studies ${ }^{8,9}$. We generated $128 \mathrm{~Gb}$ of mapped sequencing data from 13 flowcells (12 minION and 1 PromethION), with an N50 read length of 10,624 bp. (Table 1, Supplementary Table 2). We first compared genomic coverage of the resulting nanoNOMe data to whole genome bisulfite sequencing (WGBS) from a previous study (ENCODE accession 
ENCSR890UQO). We assessed the ability of nanoNOMe to cover regions that are poorly mappable via short reads by focusing on regions that were enriched in WGBS reads with low mapping score (10 or more reads with mapping quality $<5$ ). These regions covered $132 \mathrm{Mb}$ of the human genome, comprising of 57,982 distinct regions with average size of $2.3 \mathrm{~kb}$. The average coverage of high mapping quality nanoNOMe reads (mapping quality>20) in these regions was $21 \mathrm{x}$, confirming that long read sequencing, and specifically nanoNOMe, is able to cover these regions of low mappability. As an example, we plotted nanoNOMe and WGBS sequencing data in the region surrounding a SINE element, AluSP in chr5:70,903,500-70,904100 (Fig. 2b). We observed that the long reads generated from nanopore sequencing provide more even coverage across the region and stretch through the repetitive element, allowing us to measure methylation in and around the entire repetitive element.

We next assessed the performance of nanoNOMe in resolving nucleosome occupancy around CTCF binding sites as done by Kelly, et. al. ${ }^{5}$. We used CTCF binding sites determined by conserved CTCF-binding motifs ${ }^{10}$ that were $>2 \mathrm{~kb}$ away from transcription start sites and experimentally shown to be bound by CTCF in GM12878. We generated aggregate plots of methylation and chromatin accessibility relative to these CTCF binding sites (Fig. 2c). The methylation and DNA accessibility agreed with gold standard methods (WGBS and MNase-seq, respectively) from previous studies (Fig. 2c, ENCODE accession ENCSR890UQO and ENCSR000CXP). Specifically, we observed that both chromatin accessibility and methylation demonstrate an oscillation in aggregate methylation, propagating from the center of the CTCF binding sites. The distance between the peaks was $\sim 180 \mathrm{bp}$, corresponding to the typical spacing of mononucleosomes and linker DNA observed near CTCF sites.

\section{NanoNOMe reveals allele-specific patterns of methylation and nucleosome positioning}

We next explored the applicability of long-reads generated from nanoNOMe in detecting patterns of the epigenetic features. Using the epigenetic features encoded on long sequences of reads, we can observe patterns of these features along the length of the reads, e.g. positioning of multiple nucleosomes on single strands of DNA by oscillation of $\mathrm{GpC}$ methylation. Using the bisulfite mode on IGV to show GpC methylation (chromatin accessibility), we can view accessibility over the length of long reads at single-read resolution. An exemplar region demonstrating nucleosome occupancy at a CTCF binding site is shown in Fig. 3a. However, the inherent heterogeneity of the chromatin due to the dynamic nature of nucleosome positioning is directly translated to the single-read data, making it difficult to observe patterns of epigenetic features ${ }^{11}$. The biological heterogeneity of DNA accessibility is further compounded by errors associated with the enzymatic methylation, such as imperfect methylation efficacy, non-specific methylation, and dissociation of nucleosomes in a small fraction of DNA during lysis. In order to resolve patterns of methylation and DNA accessibility on single-read resolution, we have to account for the heterogeneity and noise. To that end, we focused on co-occurrences of methylated or unmethylated cytosine on each read, where the co-occurrence is defined by same type of event (methylated or unmethylated) being observed at two distinct positions on a given read (see Methods). Consolidating the co-occurrence across reads in a given region, we 
found that patterns of read-level nucleosome positioning across the length of reads can be resolved using a co-occurrence matrix (Fig. 3b). Because this analysis measures the relationship of (un)methylated cytosine between positions on individual reads, the peaks in the heatmap highlight locations of nucleosome positioning and distances between them, whereas the average plot only indicates that nucleosomes were present at the troughs without any relationship to other troughs along the region.

We then used the ability of nanopore sequencing to phase reads into haplotypes ${ }^{12}$ to compare the patterns of chromatin accessibility and methylation between maternal and paternal reads (Fig. 3c). Using existing deep coverage data on GM12878 and both parents ${ }^{13}$, we selected heterozygous SNPs belonging to either the maternal or paternal allele. Because nanopore sequencing generates long reads, each read has a greater chance of encountering a heterozygous SNP which can be used to phase the reads into maternal or paternal origin. As an example, we examined the promoter for PEG10 (Paternally expressed gene 10), which is known to be expressed only from the paternal allele ${ }^{14}$. At the PEG10 promoter, we noted a defined region that largely is unmethylated in the paternal allele while being heavily methylated in the maternal allele. We also observe allele specific chromatin accessibility in the promoter-proximal gene body of PEG10, with the paternal allele showing a region of consistently accessible while the maternal allele remains inaccessible (Fig. 3c). We used the HOMER (Hypergeometric Optimization of Motif EnRichment) suite of tools ${ }^{15}$ to examine this region of differential accessibility, and revealing that this region is dense in zinc-finger binding motifs, notably the Zn-finger transcription factors KLF5 and KLF14. Members of the KLF family are known to exert both activating and inhibitory activity through chromatin remodeling and recruitment of co-activator or co-repressors ${ }^{16}$, and therefore this region of increased accessibility on the paternal allele may highlight a regulatory element for this imprinted gene PEG10. Interestingly, both of these observations for PEG10 had been predicted by Fang et al ${ }^{17}$, using computational methods to mine short-read bisulfite sequencing data. Their analysis suggested allele specific methylation at the promoter for PEG10 as well as the presence of intragenic regulatory elements on this gene.

\section{Epigenome and Gene Expression}

We also explored the relationship between epigenetic states and gene expression. Upon generating the metaplots with respect to distance to TSS of annotated genes and stratifying the measurements based on GM12878 gene expression quartiles (ENCODE accession ENCSR843RJV), we observed that chromatin and DNA methylation show shifts in signal depending on expression, where endogenous methylation decreases at promoter regions with increasing expression and accessibility increases with increasing expression (Supplementary Fig. 2). To observe the epigenetic states for each gene and measure how the two features are directly related with respect to gene promoters, we calculated the average endogenous methylation and accessibility in individual promoter regions (400 bp window around transcription start sites) (Fig. 4a). We observed that promoters of genes with low expression tend to be highly methylated with low accessibility, and with increasing expression, the cluster shifted first to lower methylation and low accessibility, then to low methylation and higher accessibility. These 
results suggest that a combination of accessibility and methylation may be more useful to understand gene regulation than either independently.

Next, we explored the applicability of nanoNOMe on differential epigenetics analysis by performing nanoNOMe on three well-characterized breast cell lines: MCF-7 (luminal breast carcinoma, ER+/PR+/HER2-) and MDA-MB-231 (basal breast carcinoma, ER-/PR-/HER2-) as two subtypes of breast cancer, and MCF-10A (fibrocystic disease) as the normal baseline subtype (Table 1$)^{18,19}$. We achieved $>20 x$ whole genome coverage of nanoNOMe data per cell line. Comparison of epigenetic states on promoter regions of differentially expressed genes revealed that a decrease in endogenous methylation coupled with an increase in accessibility is reflective of an increase in transcription; while an increase in methylation coupled with a decrease in accessibility is correlated with a decrease in transcription (Supplementary Fig. 3). In all three comparisons, higher methylation and lower accessibility favors decrease in expression (under-expression), and lower methylation and higher accessibility favors increase in expression (over-expression).

We then examined SLC16A1, one of the genes down-regulated in the cancer cell lines, that also exhibited differential methylation and chromatin state(Fig 4b,c). The methylation frequency confirms that the promoter region of SLC16A1 is largely unmethylated in MCF-10A and methylated in MCF-7 and MDA-MB-231. The single-read data accurately captures the cell-to-cell variability in methylation, where a few of the reads in MCF-10A exhibit methylation across the entire promoter region even when the vast majority of reads are unmethylated. We can clearly observe the erosion of the $3 \mathrm{~kb}$ unmethylated region from the normal (MCF10A) to the cancer (MCF-7) to the aggressive cancer (MDA-MB-231) cell line, all on individual reads. Turning to chromatin accessibility, MCF-10A had a relatively wide region of accessible chromatin around the TSS whereas MCF-7 had a narrow window and MDA-MB-231 is completely inaccessible. Furthermore, the read-level co-occurrence revealed that MCF-7 had a strong frequency of co-occurrence of inaccessibility $-1000 \mathrm{bp}$ upstream and downstream of the TSS, suggesting blocking of transcription by occupancy up and downstream of the TSS, such as chromatin looping, whereas in MDA-MB-231, the down-regulation occurred by total occupancy of the TSS (Fig. 4d)

\section{Structural Variations and the Epigenome}

We also detected structural variations and compared nanoNOMe patterns at these sites across the three breast cell lines(Supplementary Table 3 ) $^{20,21}$. We called a total of $25,882 \mathrm{SV}$ s across all three breast lines and compared these using SURVIVOR ${ }^{21}$. The most abundant variant type were deletions $(13,974)$ followed by insertions $(10,127)$. The majority of the SVs were singletons (53.8\%) with $28.3 \%$ overlapping over two samples and $17.8 \%$ all three samples. The majority of genes $(67.36 \%)$ impacted by SVs were from deletions.

Selecting just SVs that occur only in the cancer cell lines (MCF-7 and MDA-MB-231) but not in the normal breast cell line (MCF-10A), we examined the epigenetic state of the regions flanking breakpoints. We found that regions flanking the breakpoints of structural variations do not 
exhibit consistent epigenetic characteristics in these cell lines, suggesting that structural variations have complex epigenetic consequences, dependent on more factors than the type of the variant (Supplementary Fig. 3). For example, in a 4,500 bp homozygous deletion in chr19 of MCF-7 both flanking regions are strongly hypermethylated as compared to the normal cell lines while MDA-MB-231 has milder hypermethylation. (Fig. 5a). The read-level co-occurrence chromatin occupancy in MCF-7 showed that the regions around the deletion are less frequently coordinated but instead oscillate in occupancy, indicating protection of the deletion by positioning of nucleosomes around the breakpoints. In MDA-MB-231 the entire flanking region is marked by coordinated inaccessibility (Fig. 5b).

\section{DISCUSSION}

We have demonstrated a method to examine endogenous methylation and chromatin accessibility on long fragments of DNA. Leveraging long reads we can measure allele specific methylation and chromatin profiles. We have also shown that because nanopore sequencing reads span multiple nucleosomes, nucleosome occupancy on single reads can be observed and the frequency of nucleosome positioning can be determined to observe consistency and patterns of nucleosome positioning. Using existing expression data for GM12878, we evaluated how chromosome accessibility and cytosine methylation were related to gene transcription. We found that these features were able to explain part of the differences in gene transcription, with the overall trend of the most highly expressed genes demonstrated the most accessible promoters with the lowest amount of CpG methylation. Extending our studies into breast cell lines, we compared the epigenetic state of the promoters of differentially expressed genes and found that focusing on read-level data allowed us to discern patterns of nucleosome occupancy. Further, we can observe epigenetic states of alleles with structural variants, allowing combined measurement of large genetic mutation and epigenetic state with the same assay.

However, this method is still limited, in part by using the same methylation mark that already exists in mammalian cells, 5-methylcytosine. Moving forward, we can take advantage of other methyltransferases, e.g. EcoGII which methylates adenine to N6-methyladenine. Such a technique could also provide a "multi-color" measurement, allowing further aspects of the epigenome to be integrated on the same molecule. Others have already leveraged this methyltransferase fused to lamin protein ${ }^{22}$ to explore nuclear architecture but are limited to immunoprecipitation based sequencing, precluding single molecule resolution. With further training and development, we may be able to leverage exogenous labeling with nanopore sequencing to store information about the cell state on the DNA, then sequencing it to gain long-range, phased information.

\section{METHODS}

\section{GpC methylation model generation for nanopolish}

Along with the $\mathrm{GpC}$ methylation model, the $\mathrm{CpG}$ methylation model was also regenerated to ensure the validity of the method for model generation. Genomic DNA from E. coli K12 MG1655 
(ATCC 700926DQ) and genomic DNA from GM12878 lymphoblast cell line (Coriell Institute) were first sheared to an average fragment size of $8 \mathrm{~kb}$ using g-tubes (Covaris Cat. 520079). The fragmented DNA was PCR amplified to generate unmethylated DNA using the first steps of low input ligation kit SQK-LWP001 (ONT). Briefly, samples were end-repaired, deoxyadenosine(dA)-tailed, and ligated to amplification adaptors, followed by 11 cycles of PCR amplification. The resulting unmethylated, sheared DNA was methylated with M. SssI (NEB Cat. M0226) for CpG methylation or M. CviPI (NEB Cat. M0227) for GpC methylation, or both enzymes for $\mathrm{CpG}+\mathrm{GpC}$ methylation. Two cycles of 4-hour methylation were performed for each sample, and for each cycle of treatment S-adenosylmethionine (SAM) and the enzyme were replenished at the 2 hour mark to maximize methylation levels.

Validation of DNA methylation by bisulfite sequencing

Near-complete methylation in the training samples (E. coli) and testing samples (GM12878) were validated by performing whole genome bisulfite sequencing on the Illumina MiSeq platform. NEBnext Ultra library preparation kit (NEB Cat. E7370) and Zymo EZ DNA methylation-lightning kit (Zymo Cat. D5030) were used to generate the bisulfite sequencing libraries. Briefly, DNA from each sample was shared to $300 \mathrm{bp}$ fragments using Bioruptor Pico (Diagenode), followed by end-repair and dA-tailing. Methylated universal adaptor (NEB Cat. E7535) was ligated using the Blunt/TA ligase from the kit. The adaptor-ligated samples were bisulfite-converted, quenched, and cleaned-up before PCR amplification with multiplexing primers and uracil-tolerant Taq polymerase (KAPA HiFi Uracil+ (Roche Cat. KK2801)). The resulting DNA sequencing library was sequenced on an Illumina MiSeq device using a V2 300-cycle chemistry.

The resulting data was analyzed using Bismark version $0.19 .0^{23}$. After alignment, PCR duplicates were removed using Picard tools MarkDuplicates module (http://broadinstitute.github.io/picard/). Reads were truncated at the 3 ' end to a max length of 50 bp to minimize any methylation bias at 3' ends of reads associated with the low complexity bisulfite converted libraries. The total number of methylated cytosine residues and unmethylated cytosine residues were counted to calculate methylation percentages of the samples.

\section{$\underline{\text { Cell culture }}$}

GM12878 lymphoblast cells were obtained from Coriell Institute and MCF-10A, MCF-7, and MDA-MB-231 breast cells were obtained from ATCC. GM12878 were grown in RPMI 1640 medium (Gibco Cat. 11875119) supplemented with 15\% fetal bovine serum (FBS, Gibco Cat. 26140079) and 1\% penicillin streptomycin (P/S, Gibco Cat. 15140122). MCF-10A were grown in in DMEM F-12 medium (Gibco Cat. 11320033) supplemented with 5\% horse serum (Gibco Cat. 16050122), $10 \mu \mathrm{g} / \mathrm{mL}$ human insulin (Sigma Aldrich Cat. 19278), $20 \mathrm{ng} / \mathrm{mL}$ hEGF (Gibco Cat. PHG0311L), $100 \mathrm{ng} / \mathrm{mL}$ Cholera toxin (Sigma Aldrich Cat. C8052), $0.5 \mu \mathrm{g} / \mathrm{mL}$ Hydrocortisone (Sigma Aldrich Cat. H0135), and 1\% P/S. MCF-7 and MDA-MB-231 were grown in DMEM (Gibco Cat. 11965118) supplemented with $10 \%$ FBS and $1 \%$ P/S. 


\section{Nucleosome footprinting via GpC methyltransferase}

NOMe-seq was performed to the cells with adjustments for nanopore sequencing. Cells were collected by trypsinization, then nuclei were extracted by incubating in resuspension buffer (100 $\mathrm{mM}$ Tris- $\mathrm{Cl}, \mathrm{pH} 7.4,100 \mathrm{mM} \mathrm{NaCl}, 30 \mathrm{mM} \mathrm{MgCl}_{2}$ ) with $0.25 \% \mathrm{NP}-40$ for 5 minutes on ice. Intact nuclei were collected by centrifugation for 5 minutes at $500 \mathrm{xg}$ at $4{ }^{\circ} \mathrm{C}$. Nuclei were subjected to a methylation labeling reaction using a solution of of $1 \mathrm{x}$ M. CviPI Reaction Buffer (NEB), 300 $\mathrm{mM}$ sucrose, $96 \mu \mathrm{M}$ S-adenosylmethionine (SAM; New England Biolabs, NEB), and $200 \mathrm{U} \mathrm{M}$. CviPI (NEB) in $500 \mu \mathrm{L}$ volume per 500,000 nuclei. The reaction mixture was incubated in $37^{\circ} \mathrm{C}$ with shaking on thermomixer at 1,000 rpm for 15 minutes. SAM was replenished at $96 \mu \mathrm{M}$ at 7.5 minutes into the reaction. The reaction was stopped by addition of equal volume of stop solution (20 mM Tris-Cl, pH 7.9, $600 \mathrm{mM} \mathrm{NaCl}, 1 \%$ SDS, 10 mM disodium EDTA). Samples were treated with proteinase $\mathrm{K}(\mathrm{NEB})$ at $55{ }^{\circ} \mathrm{C}$ for $>2$ hours, and DNA was extracted via pheol:chloroform extraction and ethanol precipitation. After proteinase $\mathrm{K}$ treatment, and in all following steps, samples were handled with care using large orifice pipette tips to avoid excessive fragmentation of DNA.

\section{Nanopore sequencing}

Purified gDNA was prepared for nanopore sequencing following the protocol in the genomic sequencing by ligation kit LSK-SQK108 (ONT). Samples were first sheared to $\sim 10 \mathrm{~kb}$ using G-tubes (Covaris): by centrifuging 2-3 $\mu \mathrm{g}$ of unfragmented gDNA at 5,000x g for 1 minute, then inverting the tube and centrifuging again. We sheared the DNA to $10 \mathrm{~kb}$ because it produces long fragments of DNA while maximizing the yield of nanopore sequencing. Shearing to larger sizes or unsheared DNA may be used to maximize the length of sequenced reads, with the caveat that sequencing yield will drop. The sheared samples were end-repaired and dA-tailed using NEBnext Ultra II end-repair module (NEB), followed by clean-up using $1 \mathrm{x}$ v/v AMPure XP beads (Beckman Coulter). Sequencing adaptors, comprised of leader adaptor DNA and motor proteins, were ligated to the end-prepared DNA fragments using Blunt/TA Ligase Master Mix (NEB), followed by clean-up using $0.4 \mathrm{x}$ v/v AMPure XP beads and sequencing kit reagents. $>400 \mathrm{ng}$ of adaptor ligated samples per flow cell were loaded onto FLO-MIN106 or PRO-002 flowcells and run o n MinION Mk1b, GridION, or PromethION sequencers for up to 72 hours.

\section{Training nanopolish methylation calling for $\mathrm{GpC}$ methylation}

The nanopore sequencing data from $\mathrm{GpC}$ methylated $E$. coli gDNA was used to generate the methylation model with the newest version of nanopolish (v. 0.11.0). This version has updated calculations to improve numerical stability for large datasets. We used the unmethylated and GpC methylated GM12878 gDNA as truth sets to generate the ROC curves and validate the model.

\section{Data preprocessing (basecalling, alignment, and methylation calling)}

Raw current signals were converted to DNA sequences using guppy version 2.1.3 (ONT), using basecalling configuration designed to reduce false-positive deletions in the resulting sequences 
24. DNA sequences were aligned to hg38 human reference genome without alternative contigs using NGM-LR ${ }^{20}$. CpG and GpC methylation were called using nanopolish version 0.11 .0 using a log-likelihood threshold of 2.5 to determine methylation states. We used Sniffles ${ }^{20}$ with default parameters to infer SVs across each sample and SURVIVOR ${ }^{21}$ merge to obtain a multi sample VCF file.

\section{Comparison of nanoNOMe with conventional methodologies}

Bisulfite sequencing data of GM12878 was obtained from ENCSR890UQO, and was processed using Bismark version 0.19.0. After alignment to hg38 reference genome, duplicate reads were removed using Picard tools MarkDuplicates module (http://broadinstitute.github.io/picard/) before further bismark processing to yield methylation frequency values. Normalized MNase-seq signals were obtained from ENCSR000CXP. Methylation frequency and normalized MNase-seq signal at regions surrounding genomic features of interest were extracted for the generation of the aggregate plots. For each genomic feature, average methylation frequency and accessibility was calculated by aggregating methylation calls with respect to distance from the feature and taking the rolling average with a window of $50 \mathrm{bp}$. Known TSS and CGI were obtained from Gencode (release v29). CTCF binding sites were determined by overlapping computationally predicted CTCF binding sites ${ }^{10}$ with conservative IDR peaks in ChIP-seq of CTCF on GM12878 (ENCODE accession ENCSR000AKB) and removing peaks that fell within $2 \mathrm{~kb}$ of known TSS.

\section{Read-level methylation visualization}

The bisulfite view setting of IGV was used to visually compare methylation states between samples. In order for this software to function properly, we converted all non-methylated cytosines to thymines (as would occur during bisulfite conversion). All cytosines that nanopolish called as methylated (in either $\mathrm{CpG}$ or $\mathrm{GpC}$ context) were kept as cytosines. This permitted inspection of endogenous methylation as well as chromatin accessibility using IGV.

To observe patterns of DNA methylation and accessibility in the presence of biological heterogeneity and technical variability, co-occurrence of methylated/unmethylated cytosine is calculated across reads that map to the genomic region of interest. Co-occurrence, $c$, is defined by the same event, $M$ (methylated or unmethylated), occurring on two separate binned locations, i and $\mathrm{j}$, along a given read :

$$
c_{i j}= \begin{cases}1, & \text { if } M_{i}==M_{j} \\ 0, & \text { otherwise }\end{cases}
$$

After calculating the co-occurrence for each pair of coordinates for each read, the counts are piled up to determine the frequency of co-occurrence as a measure of how often reads have the same events occurring between the positions $i$ and $j$. The resulting matrix of co-occurrence 
pileup is normalized by the maximum count, and plotted as a 2-dimensional heatmap to visualize the patterns (see accessions for code availability).

\section{Haplotype Assignment and Allele-Specific Methylation Analysis}

We obtained genotype information for GM12878 from existing phased Illumina platinum genome data generated by deep sequencing of the cell donors' familial trio ${ }^{13}$. The bcftools package was used to filter for only variants that are heterozygous in GM12878. We then used the SnpEff ${ }^{25}$ variant annotation and effect prediction tool was used to associate gene names to each read. Starting with aligned reads, we used the extractHAIRS utility of the haplotype-sensitive assembler HapCUT2 ${ }^{26}$ to identify reads with allele-informative variants. For allelic assignment, we required a read to contain at least two variants, and required that greater than $75 \%$ of identified variants agreed on the parental allele of origin -- this stringent threshold was selected to reduce the chances of incorrect assignment from nanopore sequencing errors. Through this approach each read was annotated as maternal, paternal or unassigned.

\section{Accessions}

NanoNOMe data of GM12878, MCF-10A, MCF-7, and MDA-MB-231 are available at NCBI Bioproject ID PRJNA510783 (http://www.ncbi.nlm.nih.gov/bioproject/510783). Source code is available at https://github.com/timplab/nanoNOMe.

\section{Acknowledgements}

We thank K. D. Hansen for helpful discussions. This study was supported by National Human Genome Research Institute (NHGRI project 5R01HG009190).

\section{References}

1. Hansen, K. D. et al. Increased methylation variation in epigenetic domains across cancer types. Nat. Genet. 43, 768-775 (2011).

2. Landan, G. et al. Epigenetic polymorphism and the stochastic formation of differentially methylated regions in normal and cancerous tissues. Nat. Genet. 44, 1207-1214 (2012).

3. Boyle, A. P. et al. High-resolution mapping and characterization of open chromatin across the genome. Cell 132, 311-322 (2008).

4. Buenrostro, J. D., Giresi, P. G., Zaba, L. C., Chang, H. Y. \& Greenleaf, W. J. Transposition of native chromatin for fast and sensitive epigenomic profiling of open chromatin, DNA-binding proteins and nucleosome position. Nat. Methods 10, 1213-1218 (2013). 
5. Kelly, T. K. et al. Genome-wide mapping of nucleosome positioning and DNA methylation within individual DNA molecules. Genome Res. 22, 2497-2506 (2012).

6. Simpson, J. T. et al. Detecting DNA cytosine methylation using nanopore sequencing. Nat. Methods 14, 407-410 (2017).

7. Rand, A. C. et al. Mapping DNA methylation with high-throughput nanopore sequencing. Nat. Methods 14, 411-413 (2017).

8. Zook, J. M. et al. Extensive sequencing of seven human genomes to characterize benchmark reference materials. Sci Data 3, 160025 (2016).

9. ENCODE Project Consortium. An integrated encyclopedia of DNA elements in the human genome. Nature 489, 57-74 (2012).

10. Ziebarth, J. D., Bhattacharya, A. \& Cui, Y. CTCFBSDB 2.0: a database for CTCF-binding sites and genome organization. Nucleic Acids Res. 41, D188-94 (2013).

11. Lai, W. K. M. \& Pugh, B. F. Understanding nucleosome dynamics and their links to gene expression and DNA replication. Nat. Rev. Mol. Cell Biol. 18, 548-562 (2017).

12. Gigante, S. et al. Using long-read sequencing to detect imprinted DNA methylation. doi:10.1101/445924

13. Eberle, M. A. et al. A reference data set of 5.4 million phased human variants validated by genetic inheritance from sequencing a three-generation 17-member pedigree. Genome Res. (2016). doi:10.1101/gr.210500.116

14. Ono, R. et al. A retrotransposon-derived gene, PEG10, is a novel imprinted gene located on human chromosome 7q21. Genomics 73, 232-237 (2001).

15. Heinz, S. et al. Simple combinations of lineage-determining transcription factors prime cis-regulatory elements required for macrophage and B cell identities. Mol. Cell 38, 576-589 (2010). 
16. McConnell, B. B. \& Yang, V. W. Mammalian Krüppel-like factors in health and diseases. Physiol. Rev. 90, 1337-1381 (2010).

17. Fang, F. et al. Genomic landscape of human allele-specific DNA methylation. Proc. Natl. Acad. Sci. U. S. A. 109, 7332-7337 (2012).

18. Holliday, D. L. \& Speirs, V. Choosing the right cell line for breast cancer research. Breast Cancer Res. 13, 215 (2011).

19. Messier, T. L. et al. Histone H3 lysine 4 acetylation and methylation dynamics define breast cancer subtypes. Oncotarget 7, 5094 (2016).

20. Sedlazeck, F. J. et al. Accurate detection of complex structural variations using single-molecule sequencing. Nat. Methods 15, 461-468 (2018).

21. Jeffares, D. C. et al. Transient structural variations have strong effects on quantitative traits and reproductive isolation in fission yeast. Nat. Commun. 8, 14061 (2017).

22. Sobecki, M. et al. MadID, a Versatile Approach to Map Protein-DNA Interactions, Highlights Telomere-Nuclear Envelope Contact Sites in Human Cells. Cell Rep. 25, 2891-2903.e5 (2018).

23. Krueger, F. \& Andrews, S. R. Bismark: a flexible aligner and methylation caller for Bisulfite-Seq applications. Bioinformatics 27, 1571-1572 (2011).

24. Jain, M. et al. Nanopore sequencing and assembly of a human genome with ultra-long reads. Nat. Biotechnol. 36, 338-345 (2018).

25. Cingolani, P. et al. A program for annotating and predicting the effects of single nucleotide polymorphisms, SnpEff: SNPs in the genome of Drosophila melanogaster strain w1118; iso-2; iso-3. Fly 6, 80-92 (2012).

26. Edge, P., Bafna, V. \& Bansal, V. HapCUT2: robust and accurate haplotype assembly for diverse sequencing technologies. Genome Res. 27, 801-812 (2017). 


\section{FIGURES / TABLES}

\section{Figures}

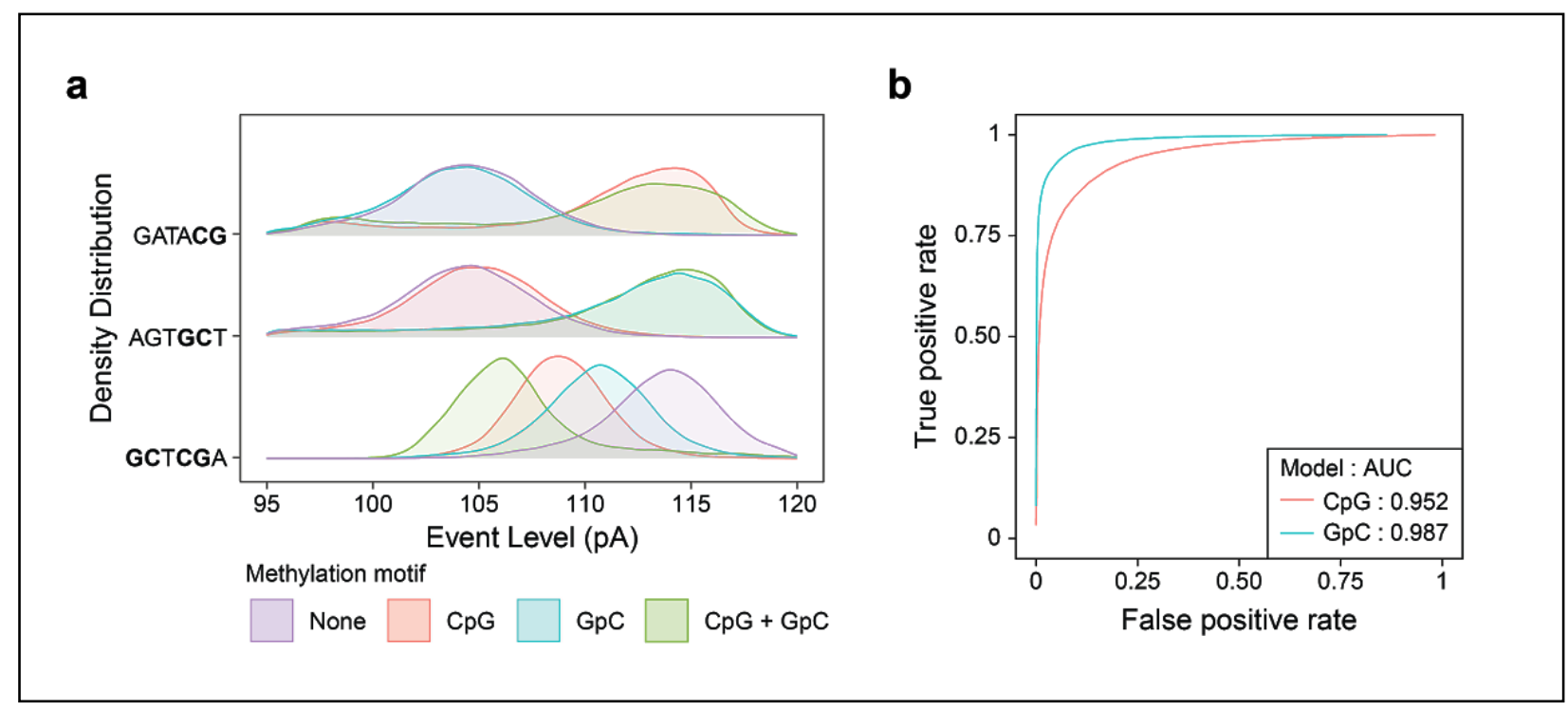

Figure 1. Nanopore sequencing accurately detects GpC methylation. (a) Event level current density distribution for select k-mers having $\mathrm{CpG}, \mathrm{GpC}$, and both $\mathrm{CpG}$ and $\mathrm{GpC}$ motif for E. Coli genomic DNA with no methylation, $\mathrm{CpG}, \mathrm{GpC}$, and both $\mathrm{CpG}$ and $\mathrm{GpC}$ methylation. (b) ROC curve for a range of thresholds for methylation detection on control samples (GM12878 genomic DNA modified with CpG and GpC methylation). 


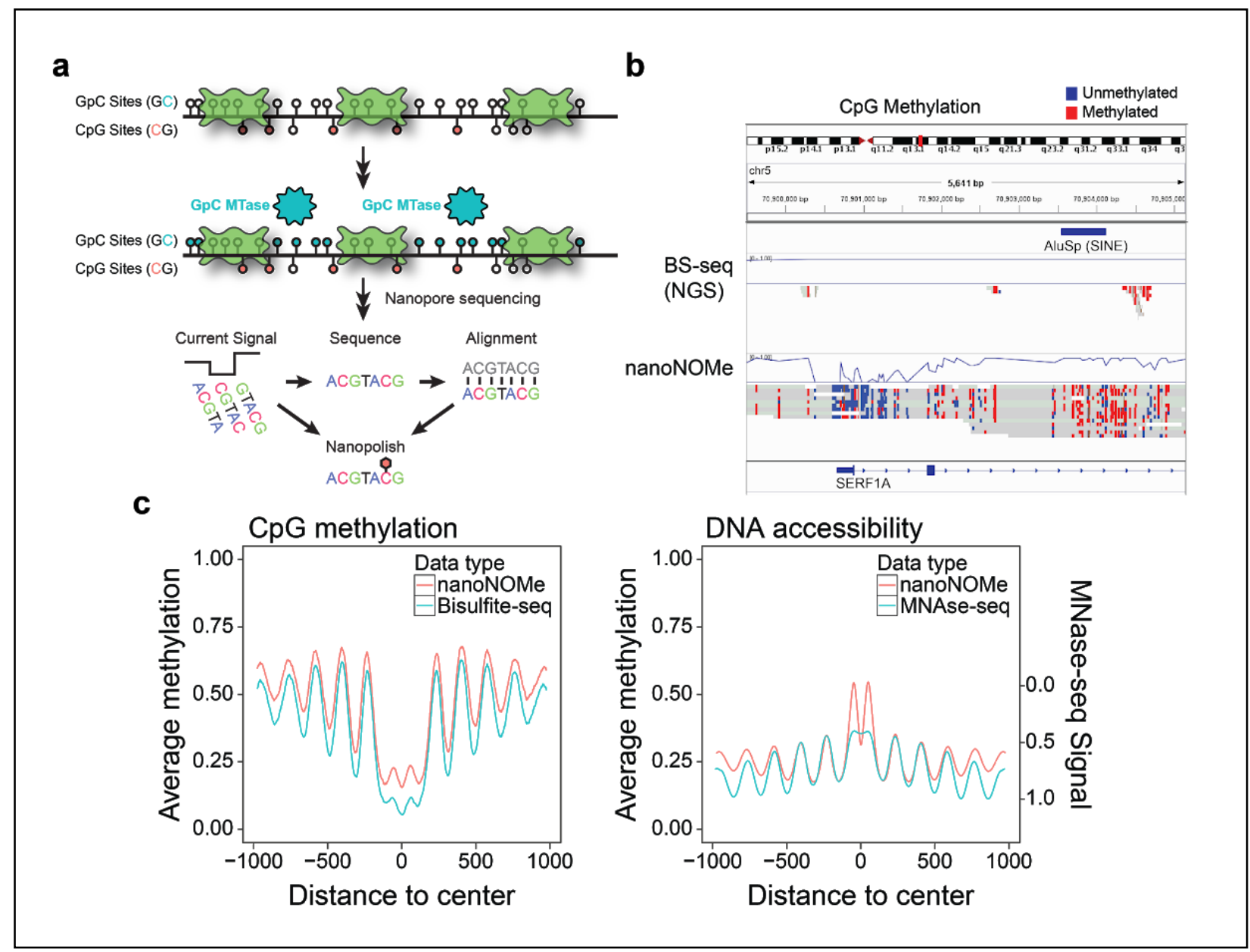

Figure 2. NanoNOMe can measure endogenous DNA methylation and accessibility. (a) nanoNOMe uses exogenous methylation of accessible DNA by GpC methyltransferase, followed by nanopore sequencing and nanopolish methylation detection. (b) Using bisulfite mode of IGV to visualize methylation on nanopore sequencing reads versus bisulfite sequencing. Note gaps in coverage in highly repetitive region. (c) Aggregate plots of endogenous methylation (left) and chromatin accessibility (right) from nanoNOMe (red) and conventional assays (BS-seq and MNase-seq, blue) centered around CTCF binding sites. 




Figure 3. Long single read chromatin and methylation patterns. (a) GpC methylation (accessibility) of a CTCF binding site, both average frequency (top) and individual reads (bottom). (b) Co-occurrence of accessibility and inaccessibility ( $\mathrm{GpC}$ ) across the lengths of single reads and consolidating co-occurrences across all reads in the region resolves patterns such as nucleosome occupancy. (c) Reads mapped to the PEG10 gene region were separated based on alleles, showing that the reads from paternal, expressed, allele are unmethylated $(\mathrm{CpG})$ at the promoter and open $(\mathrm{GpC})$ in the gene body. 


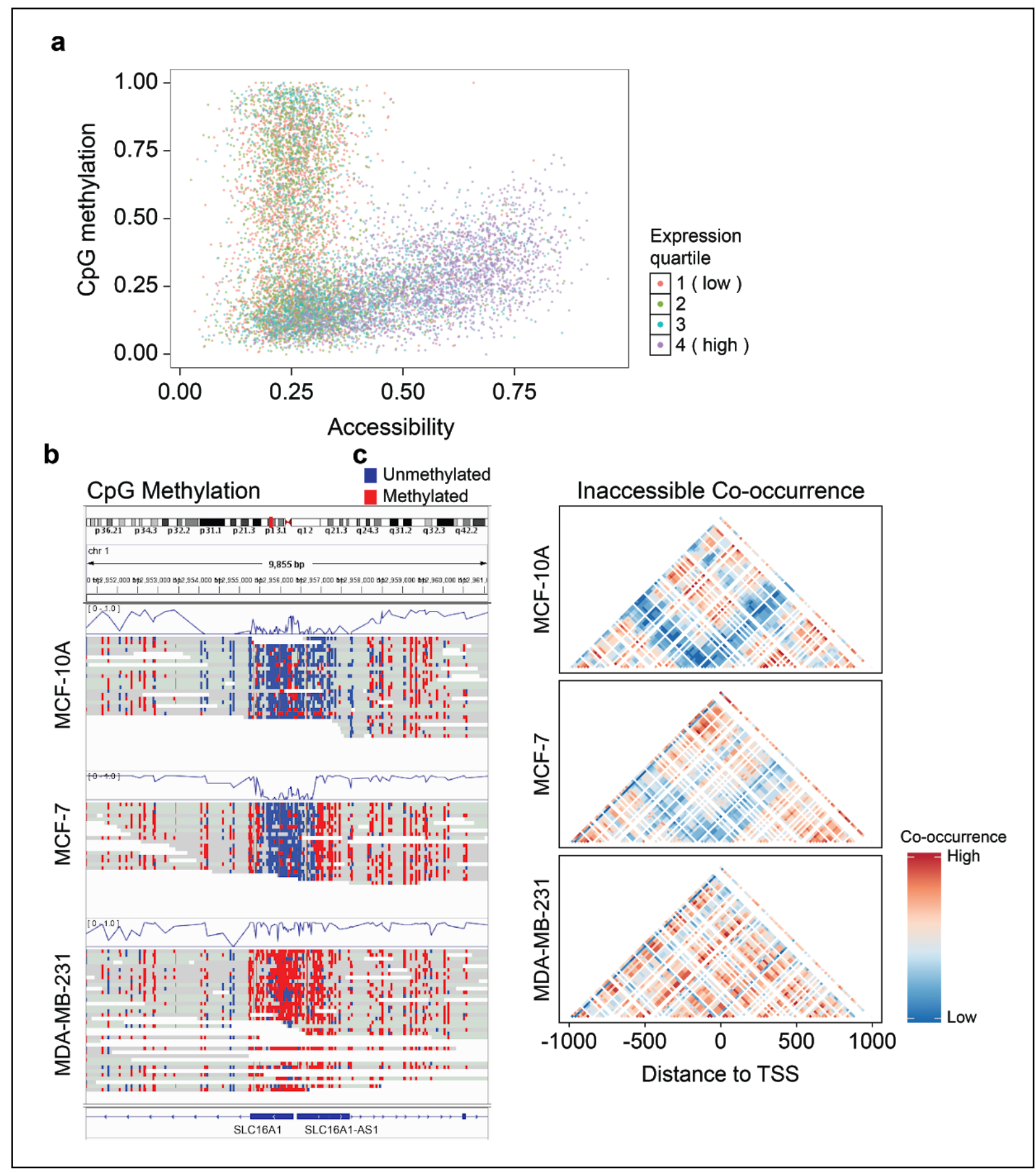

Figure 4. Epigenetic state correlation with gene expression (a) Scatter plots of endogenous methylation versus accessibility color-coded by expression quartile in GM12878. Each dot in the scatter plot represents the methylation and chromatin state of a promoter region of a gene (200 bp +/- TSS) (c) IGV view of endogenous methylation surrounding SLC16A1 shows the differential methylation around the promoter region of the gene and (d) co-occurrence plot of accessibility and inaccessibility showing increased co-occurrence of chromatin inaccessibility around the promoter. 


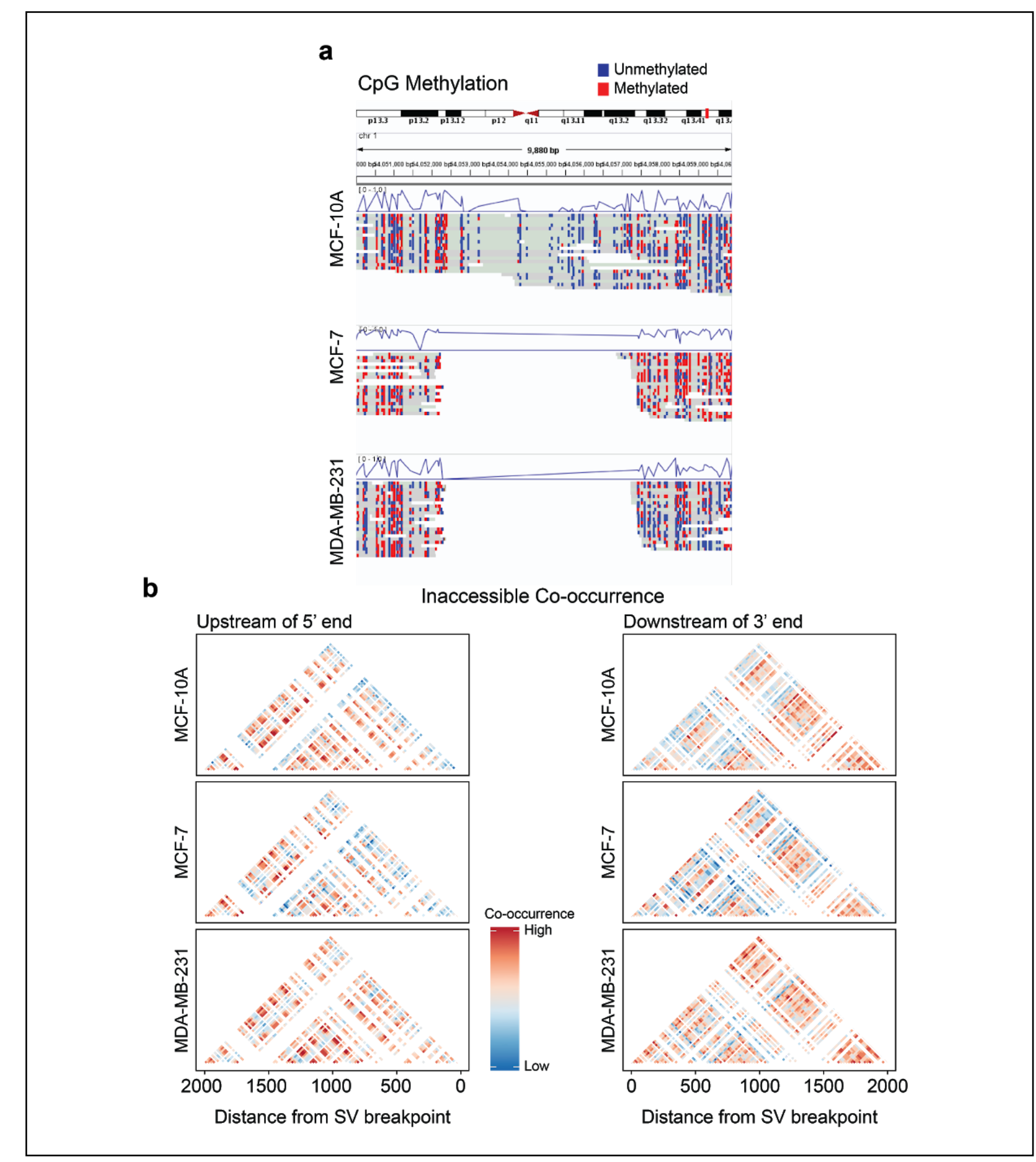

Figure 5. Epigenetic state comparison of homozygous deletion present only in MCF-7 and MDA-MB-231. (a) A deletion in IGV view shows that the flanking regions around the deletion in MCF-7 are hypermethylated compared to MCF-10A while MDA-MB-231 is not, and (b) co-occurrence matrix of inaccessible events indicates MCF-7 has less consistent inaccessibility (more blue) and more nucleosome occupancy around the breakpoints (oscillation in color) whereas MDA-MB-231 is marked by increased inaccessibility up and downstream. 
bioRxiv preprint doi: httos://doi org/101101/504993: this version posted Februarv 2 2019. The copvriaht holder for this preprint (which was not certified by peer review) is the author/funder, who has granted bioRxiv a license to display the preprint in perpetuity. It is made available under aCC-BY 4.0 International license.

\section{Tables}

\begin{tabular}{|c|c|c|c|c|c|c|c|}
\hline Cell & $\begin{array}{l}\text { Number of } \\
\text { flowcells }\end{array}$ & $\begin{array}{l}\text { Number of raw } \\
\text { reads (M) }\end{array}$ & $\begin{array}{l}\text { Total raw bases } \\
\text { (Gb) }\end{array}$ & $\begin{array}{l}\text { Aligned reads } \\
\text { (M) }\end{array}$ & $\begin{array}{l}\text { Aligned } \\
\text { bases (Gb) }\end{array}$ & Coverage & N50 length \\
\hline GM12878 & $12+1$ Plon $^{*}$ & 20.9 & 148.5 & 16.6 & 127.6 & 39 & 10,624 \\
\hline MCF-10A & 9 & 9.4 & 81.6 & 7.7 & 72.4 & 22 & 11,501 \\
\hline MCF-7 & 11 & 9.0 & 76.8 & 7.5 & 69.1 & 21 & 13,025 \\
\hline MDA-MB-231 & 9 & 8.0 & 82.4 & 7.0 & 74.9 & 23 & 13,507 \\
\hline
\end{tabular}

Table 1. Sequencing statistics of nanoNOMe data. NanoNOMe was performed on four cell lines using multiple runs of MinION, GridION, or PromethION sequencing and pooled to generate one data set per cell line. 